\title{
Einstein's gravitation is Einstein-Grossmann's equations
}

\author{
Alfonso Leon Guillen Gomez \\ Independent scientific researcher, Bogota, Colombia \\ E-mail: aguillen@gmx.net
}

\begin{abstract}
While the philosophers of science discuss the General Relativity, the mathematical physicists do not question it. Therefore, there is a conflict. From the theoretical point view "the question of precisely what Einstein discovered remains unanswered, for we have no consensus over the exact nature of the theory's foundations. Is this the theory that extends the relativity of motion from inertial motion to accelerated motion, as Einstein contended? Or is it just a theory that treats gravitation geometrically in the spacetime setting?". "The voices of dissent proclaim that Einstein was mistaken over the fundamental ideas of his own theory and that their basic principles are simply incompatible with this theory. Many newer texts make no mention of the principles Einstein listed as fundamental to his theory; they appear as neither axiom nor theorem. At best, they are recalled as ideas of purely historical importance in the theory's formation. The very name General Relativity is now routinely condemned as a misnomer and its use often zealously avoided in favour of, say, Einstein's theory of gravitation What has complicated an easy resolution of the debate are the alterations of Einstein's own position on the foundations of his theory", (Norton, 1993) [1]. Of other hand from the mathematical point view the "General Relativity had been formulated as a messy set of partial differential equations in a single coordinate system. People were so pleased when they found a solution that they didn't care that it probably had no physical significance" (Hawking and Penrose, 1996) [2]. So, during a time, the declaration of quantum theorists: "I take the positivist viewpoint that a physical theory is just a mathematical model and that it is meaningless to ask whether it corresponds to reality. All that one can ask is that its predictions should be in agreement with observation." (Hawking and Penrose, 1996) [2] seemed to solve the problem, but recently achieved with the help of the tightly and collectively synchronized clocks in orbit frontally contradicts fundamental assumptions of the theory of Relativity. These observations are in disagree from predictions of the theory of Relativity. (Hatch, 2004a, 2004b, 2007) [3,4,5]. The mathematical model was developed first by Grossmann who presented it, in 1913, as the mathematical part of the Entwurf theory, still referred to a curved Minkowski spacetime. Einstein completed the mathematical model, in 1915, formulated for Riemann's spacetimes. In this paper, we present as of General Relativity currently remains only the mathematical model, darkened with the results of Hatch and, course, we conclude that a Einstein's gravity theory does not exist.
\end{abstract}

\section{Keywords}

General Relativity; general principle of relativity; strong equivalence principle; Mach's principle; Einstein-Grossmann equations; general covariance; Newton equations

\section{PACS}

04. General Relativity and gravitation, 04.20.-q Classical General Relativity, 04.20.Cv Fundamental problems and general formalism

\section{Council for Innovative Research}

\author{
Peer Review Research Publishing System
}

Journal: JOURNAL OF ADVANCES IN PHYSICS

Vol. 11, No. 3
www.cirjap.com, japeditor@gmail.com 


\section{Introduction.}

The Special Relativity was constructed as the first physical theory based in the Galilei principle of relativity of inertial motion, in the particular form that is Lorentz covariant. "Lorentz covariance became synonymous with satisfaction of the principle of relativity of inertial motion and the whole theory itself" (Norton, 1993) [1] .

With the discovery of the geometries non euclidean hyperbolic and elliptic, Klein, colleague of Minkowski, conducted the Erlangen program and Minkowski introduced the geometric approach into Special Relativity theory. Minkowski "proposed categorizing the new geometries by their characteristic groups of transformations. Euclidean geometry, for example, was characterized by the group of rotations, translations and reflections. The entities of the geometry were the invariants of these transformations" (Norton, 1993) [1]. The next step was pass from geometry to physics. Minkowski characterized the group of transformation under the notion of spacetime in the motion of inertial systems and "constructed the geometry associated with the Lorentz transformation covariant", "only spacetime vectors may appear in physical equations" . "From this geometric perspective, the formulation of a theory that satisfied the principle of relativity became trivial. One merely needed to formulate the theory in terms of the geometric entities of the spacetime -in effect the various types of spacetime vectors Minkowski had defined- and the theory would be automatically Lorentz covariant" (Norton, 1993) [1].

The difference between Einstein and Minkowski's approach "to the same theory and even the same formalism" is that throughout the development of both Special and General Relativity theories, Einstein's emphased "on the algebraic properties of the theory, the equations that express its laws and their behaviour under transformation, its covariance. Thus the satisfaction of the principle of relativity is established by algebraic manipulation. The equations of the theory are transformed under the Lorentz transformation and the resulting equations are shown to have preserved their form. Minkowski's emphased on the geometric properties of the theory, on those geometric entities which remain unchanged behind the transformations, its invariance. Thus Minkowski ensures satisfaction of the principle of relativity by quite different means" (Norton, 1993) [1].

During the creation of General Relativity, there were two key moments that were the formulation of a partial version called Entwurf theory and the complete work. These moments are:

1. The Entwurf theory was made between Einstein and his friend and colleague the mathematician Grossmann, after less than 9 months of work. The Entwurf theory was published, in the middle of 1913, as "Outline of a generalized theory of relativity and of a theory of gravitation" compound of the I. Physical Part of Albert Einstein and the II. Mathematical Part of Marcel Grossmann, his equations were limited generally covariant and essentially are the foundations of the General Relativity.

2. On 25 November 1915, according to his full generally covariant formulation, Albert Einstein presented at the Prussian Academy meeting in Berlin: The gravitational field equations, five days after that David Hilbert presented at a meeting of the Royal Academy of Sciences in Göttingen: "The foundations of physics" which also contained the correct field equations. Hilbert was the best germane mathematician of the epoch. "In 1915 Hilbert worked on the same problem as Einstein and needed Einstein's November 4 and 1914 papers for his theory of gravitation. It appears that Einstein's papers were crucial for the development of his theory but Hilbert also inspired Einstein in his November 11 and 18 works" (Weinstein, 2014) [6]. Both scientists were working on a theory of gravity and its equations and sought lean on the other. They recognized the intense exchange of correspondence on their progresses during October and that November, which it became daily. What did they learn one from other? It is uncertain.

Of course, the work of Einstein on gravitation is compound of the physical theory and the respective field equations.

Between 1907-1918, Einstein structured the physical theory on the gravitation as the General Relativity theory based in the three following principles:

1. Strong principle of equivalence, 1907-1912, called equivalence Einstein's principle, establishes that acceleration and gravity are equivalents.

2. Mach's principle, 1912-1917, explains the inertia of the bodies and particles as completely determined by the total mass in the universe of them.

3. General covariance, 1913-1916, that sought extends the principle of relativity, called principle general of relativity, of inertial motion, to accelerated motion.

General covariance is the fundamental principle because it is the reason for the name of General Relativity, that Einstein gave to his theory on gravitation. It implies that the laws of nature remained unchanged under all coordinate transformation, i.e. the laws of nature are independents of the coordinates. "Einstein (1918) introduces the three principles with the remark that they are 'in any case in no way independent of one another'. Thus it is not clear whether these particular two of the three principles really are independent or, if they are not, whether general covariance somehow leads to Mach's principle. Perhaps the best answer we will find is Einstein's repeated insistence that general covariance, in conjunction with a requirement of simplicity, leads us directly to General Relativity. And it is this theory that eliminates the absoluteness of the inertial system" (Norton, 1993) [1]. But, Einstein "lost completely his enthusiasm for Mach's principle, abandoning it unequivocally in his later life" (Norton, 1995) [7].

Undoubtedly, the main formal connection between theory and equations was the principle of general covariance that satisfy the field equations. Thus, between 1912 and 1915, Einstein obtained the field equations through of his struggled 
with general covariance, characterized at the following moments:

1. Field equations based in limited general covariance. Initially, Einstein concluded that the gravitational field must be expressed by the metric tensor of a curved four-dimensional Minkowski space-time. On other hand, Grossmann identified to the Riemann tensor as the only tensor, that it can obtain from the first and second derivatives of the metric tensor and to the contracted Riemann tensor for find the field equations that they would be generally covariants. However, the equations of Grossmann were fixed to limited generally covariant field equations and failed to yield the correct Newtonian limit due to that the equations of gravitation of Newton are not valid in all coordinate systems. "Only their covariance with respect to linear transformations is certain" (Einstein, 1913) [8]. After published the Entwurf theory, Einstein convinced of the physical indeterminism caused by his argument of the hole, as result of misapplication of the general covariance, he rejection it.

2. Field equations based in full general covariance. Finally, Einstein accepted the general covariance and he had to get rid of the hole argument. "He was helped by a philosophical argument due to Moritz Schlick stating that objective physical events can only be identified with the help of physically real space-time coincidences which already presuppose the existence of a gravitational field" (Renn, 2013) [9], (Engler, 2009) [10]. Thus, "Einstein beaten strongly by the advance of Hilbert, during only four critical and distressing days, after years of work, helped by Schlick and returning to Grossmann found the final field equations. The equations of Grossmann were very closed to the final equations of Einstein. Therefore there was that restrict the set of coordinate systems under consideration through the explicit stipulation of the called "coordinate conditions", satisfied by the final equations" (Norton, 1984) [11]. However, Einstein considered: "Grossmann will never claim to be co-discoverer. He only helped me to orient myself in the mathematical literature, but contributed nothing materially to the results" (Earman and Glymour, 1978) [12].

While the field equations are not appointed as erroneous, however, the validity of the principles have been discussed since its introduction, quickly Mach's principle was abandoned, and the rest has been polemicized hitherto. The consensus, 100 years later, is that the principles fail. Currently, General Relativity is supported only in his field equations. In this article it establishes that, notwithstanding, the principles from which was derived the mathematical model, evidently, they not are accepted as true without controversy, paradoxically the mathematical model yield numerous experimental predictions with great accuracy.

\section{General covariance is not general relativity.}

The point of initiation of the General Relativity was the question of whether the principle of relativity of inertial motion could be extended to uniform rectilinear accelerated motion. The strategy was link the relativity principle with covariance and equivalence principles. The result was identify relativity with covariance depending of equivalence.

According to the equivalence principle (inertial mass equivalent to gravitational mass), until 1911, understood as the uniform acceleration equivalent to homogeneous gravitational field, that was extended, from the Lorentz covariance of the Special Relativity to the limited covariance, in the Minkowski's spacetimes: first in flat spacetime (Einstein, 1907) [13], after, unreached to curved spacetime (Einstein, 1912) [14]. Neither to the general covariance in the Riemann's spacetime, formulated in November of 1915.

"The equivalence of inertial frames in classical mechanics was extended to non-mechanical processes by Einstein and became one of the two basic postulates of Special Relativity theory. In his General Relativity theory Einstein set out to generalize this principle to arbitrary frames of reference. There seems to be a consensus among philosophers of physics that he failed" (Dieks, 2005) [15]. "Einstein believed that because the laws of his General theory were generally covariant, they automatically satisfied a general principle of relativity. The reason given for this belief shows the confusion of point and coordinate transformation and of reference and coordinate systems (Earman and Glimour, 1978) [12].

When the limited general covariance, expressed by the metric tensor of a curved four-dimensional Minkowski spacetime, was passed to arbitrary transformations of any kind, that is, to the general covariance but it did not yield the correct Newtonian limit due to that the equations of gravitation of Newton are not valid in all coordinate systems. Again, the covariance was valid only under a restricted class (although broader than the Lorentz group) of linear transformations. Thus, Einstein and Grossman changed their gravitational field equations by other that were not generally covariant.

"Einstein and Grossman started with the invariant interval of Minkowski in differential form:

$d s^{2}=c^{2} d t^{2}-d x^{2}-d y^{2}-d z^{2}$

where $(x, y, z, t)$ are the space and time coordinates of an inertial frame of reference in a Minkowski spacetime. Transforming to arbitrary coordinates $x_{\mu}$ for $\mu=1, \ldots, 4,(3)$ becomes:

$d s^{2}=g_{\mu v} d x_{\mu} d x_{v}$

Einstein employed his principle of equivalence to interpret the matrix of quantities $g_{\mu v}$ that had arisen with the introduction of arbitrary coordinates. In the special case of the principle, the transformation from (3) to (4) is from an inertial coordinate system to a uniformly accelerated coordinate system. In that case, the matrix of coefficients $g_{\mu v}$ reduces to that of (3), except that $c$ now is a function of the coordinates $\left(x^{\prime}, y^{\prime}, z^{\prime}\right)$. That is, (4) becomes

$d s^{2}=c^{2}\left(x^{\prime}, y^{\prime}, z^{\prime}\right) d t^{\prime 2}-d x^{\prime 2}-d y^{\prime 2}-d z^{\prime 2}$

According to the principle of equivalence, the presence of a gravitational field was the only difference between the 
spacetime of (3') and that of Special Relativity (3). Therefore Einstein interpreted the coordinate dependent c of (3') as representing a gravitational field and, more generally, the $g_{\mu v}$ of (4) as representing a gravitational field.

Einstein and Grossman proceeded to develop essentially all the major components of the final General Relativity theory. Just one eluded them. The spacetimes represented by (3), (3') and (4) are all flat" (Norton, 1993) [1].

After published the Entwurf theory, to final 1914, Einstein, convinced of the physical indeterminism caused by his hole argument, he condemned and abandoned the general covariance. However, a year later, (November 1915), in the hard struggle with Hilbert for arrive to the equations of the General Relativity in Riemann's spacetimes, in photo finish, winning by Hilbert, Einstein was helped by the philosopher Schlick and with the point-coincidences argument, the general covariance was successfully reintroduced. Einstein finally understood that the physical properties of the points within the hole depends on the metric and, therefore, there are no such properties before specify metric.

Of other hand, "to treat the general case of the gravitational field, non-flat metrics must also be admitted and, in the final theory, the decision of which are admitted is made by the gravitational field equations (Norton,1993) [1]". Thus, the equations take the form $\mathrm{G}_{\mu \mathrm{v}}=\kappa \mathrm{T}_{\mu \mathrm{v}}$.

However, general covariance is not general relativity principle. That the physical laws do not change under an arbitrary transformation of the spacetime coordinates does not means that the principle of relativity of inertial motion was extended to any accelerated motion. "In the Lorentz covariant formulation of the Special Relativity, groups associated with covariance and relativity principles happen to coincide. With the transition to General Relativity, the covariance group grew to the general group. What Einstein missed was that the group associated with the principle of relativity did no grow with it. It shrank to the identity group. Had einstein pursued the geometric approach of Minkowski rather his own algebraic approach would have been far less likely to confuse covariance and relativity" (Norton, 1995) [7]. "Convinced by Grossman that no second order tensor expression was available to generalize Poisson's equation, Einstein produced arguments to show that general covariance was impossible for gravitational theory. Pressed by Mie about the lack of general covariance for his 1913 field equations, he produced an argument to show that covariance was of no physical importance. When after a month's furious work, Einstein finally hit on satisfactory generally covariant equations in November of 1915, he rapidly produced arguments to show that covariance is essential. Almost none of these arguments are, from either a modern or a contemporary standpoint, satisfactory; typically they confuse coordinate systems and reference frames, coordinate transformations and point transformations, covariance and space-time structure" (Earman and Glimour, 1978) [12].

"If covariance principles are not relativity principles, then what are relativity principles? New answers to this question have come repeatedly within the tradition that proposes the divorce of general covariance from a generalization of principle of relativity. We shall see that they eventually stabilize on the view that a relativity principle expresses a symmetry of the spacetime structure" (Norton, 1993) [1]. "Models $\left(M, \eta_{a b}, T_{a b}\right)$ satisfies a relativity principle associated with the Lorentz group exactly because a Lorentz transformation is a symmetry of the pair $\left(M, \eta_{a b}\right)$ which represents the background spacetime". "In General Relativity the corresponding models are $\left(M, g_{a b}, T_{a b}\right)$ for the symmetry group $\left(M, g_{a b}\right)$ is, in general, the identity group" (Norton, 1995) [7]. "Thus the physical content of the theory fixes the metrical components. But once these components are fixed, the coordinate system is fixed and no covariance transformation remains; in effect the covariance group has become the identity group and one has no relativity principle" (Norton, 1993) [1].

By 1916, it is accepted that Einstein to consistently managed to integrate general covariance with the principle of equivalence. "Einstein offered the principle of general covariance as the fundamental physical principle of his General Relativity and as responsible for extending the principle of relativity to accelerated motion. This view was disputed almost immediately with the counter-claim that the principle was no relativity principle and was physically vacuous. The disagreement persists today..." . "Special Relativity required the complete physical equivalence of all inertial coordinate systems; for Einstein, General Relativity required the complete equivalence of all coordinate systems. Einstein's principle of equivalence required the complete equivalence of a set of coordinate systems of intermediate size: inertial coordinate systems plus uniformly accelerated coordinate systems. That is, the principle sanctioned the extension of the covariance of Special Relativity beyond Lorenzt covariance but not as far as general covariance. Thus, for Einstein, the principle of equivalence was a relativity principle intermediate in range between the principle of relativity of Special Relativity and of general" (Norton, 1993) [1].

In 1917, Kretschmann said that if we accept the point coincidence argument then general covariance lacks of real physical content and no generalizes the principle of relativity due to that all the spacetime theories, including Special Relativity and Newtonian spacetime theory, can be formulated as general covariants. "By means of a purely mathematical reformulation of the equations representing the theory, and with, at most, mathematical complications conected with that reformulation, any physical theory can be brought into agreement with any, arbitrary relativity postulate, even the most general one, and this without modifying any of its content that can be tested by observation". This is the objection more important in relation to the principle of generalized relativity. "Einstein was shortly proved wrong. Cartan (1923) and Friedrichs (1927) found serviceable, generally covariant formulations of Newtonian gravitation theory. Einstein was right in so far as these generally covariant formulations were more complex than General Relativity. However Einstein was quite wrong in predicting that such formulations would not be usable practically" (Norton, 1993) [1].

In 1918, Einstein responded that between various empirically viable mathematical systems competing, from the standpoint of the absolute differential calculus the system more simpler is preferable, although, in the sense of the system that is physical possible with respect to the practically impossible systems and no merely a matter of convenience. "Let one bring Newtonian gravitational mechanics into the form of absolutely covariant equations (four-dimensional) and one will 
certainly be convinced that principle excludes this theory, not theoretically, but practically" (Einstein, 1918) [16]. The philosophical context of Einstein was the metaphysics of the than it derives, it can recognize the truth of a theory in its mathematical simplicity. Thus, the general covariance of the relativity would reach this simplicity. However, if there are two or more theories tied to its mathematical simplicity, all are true?. How to choose?. In general, in the theory on systems, Einstein would have optimized a system, but he no found a method for generalize the principle of relativity of Galilei. How can the general covariance, generalize the principle relativity?. "Relativity principles are symmetry principles; the requirement of general covariance is not a symmetry principle" (Norton, 1993) [1]. "Generally covariant formulations of prerelativistic theories (i.e., Newtonian and specially relativistic theories) are now utterly familiar in philosophical and foundational discussion. Even if it can be argued that, so formulated, the physical content of such a theory is somehow different from that of the "standard", non-covariant formulation (a claim I reject), it seems that general covariance cannot be what distinguishes General Relativity from generally covariant versions of pre-relativistic theories" (Pooley, 2010) [17]. "The requirement of general covariance, taken by itself, is even devoid of physical content. It can be salvaged as a physical principle by supplementing it with further requirements. The most popular are a restriction to simple law forms and a restriction on the additional structures that may be used to achieve general covariance. However neither supplementary condition has been developed systematically beyond the stage of fairly casual remarks" (Norton, 1993) [1].

In the long controversy on the argument of Kretschmann, Norton considers that: "The best developed of these attempts is due to Anderson and is based on the distinction of absolute from dynamical objects. General Relativity satisfies Anderson's principle of general invariance which entails that the theory can employ no non-trivial absolute objects. This principle is offered as a clearer statement of Einstein's real intentions and as giving a precise interpretation of Einstein's repeated disavowal of the absolutes of Newton's space and time" (Norton, 1993) [1]. An absolute object affects the behavior of other objects but is not affected by these objects in tum. The remaining objects are dynamical. When there are absolute objects the invariance group is the subgroup of the covariance group which leaves invariant the absolute objects. If there are no absolute objects, the invariance group and the covariance group are the same group.

"Anderson's central claim is that this symmetry group is what Einstein really had in mind when he associated the Lorentz group with Special Relativity and the general group with General Relativity. For a requirement on a symmetry group, not a covariance group is the correct way to express a relativity principle"... "The symmetry group of a generally covariant Special Relativity is the Lorentz group. Again, consider a generally covariant formulation of Newtonian spacetime theory with spacetime structures ta, $\mathrm{h}^{\alpha \beta}$ and $\nabla \alpha$, where the gravitational field is not incorporated into $\nabla \alpha$. Then these three objects are the absolute objects of the theory and their symmetry group is the Galilean group. Finally, General Relativity has no absolute objects. Its symmetry group is the general group" (Norton, 1993) [1].

"A field theory is formulated in manner invariant under passive diffs (or change of coordinates), if we can change the coordinates of the manifold, re-express all the geometric quantities (dynamical and non-dynamical) in the new coordinates, and the form of the equations of motion does not change. A theory is invariant under active diffs, when a smooth displacement of the dynamical fields (the dynamical fields alone) over the manifold, sends solutions of the equations of motion into solutions of the equations of motion". (Rovelli, 2001) [18].

Philosophers of space and time do not believe that Anderson's reasoning has vindicated Einstein's claim that the General Relativity extends the principle of relativity of Special Relativity.

"The dynamical character of the metric is irrelevant in this context and that the metric must be preserved under the theory's symmetry group. if that group is to be associated with a relativity principle. Since the metrics of general relativistic spacetimes have, in general, no non-trivial symmetries, there is no non-trivial relativity principle in General Relativity. Whatever may have been its role and place historically, general covariance is now automatically achieved by routine methods in the formulation of all seriously considered spacetime theories. The foundations of General Relativity do not lie in one or other principle advanced by Einstein. Rather, they lie in the simple assertion that spacetime is semi-Riemannian, with gravity represented by its curvature and its metric tensor governed by the Einstein field equations" (Norton, 1993) [1].

Certainly, general covariance is not the general relativity principle, neither general covariance is a principle, but mathematical property of the tensors, because the laws of physics expressed in the form of tensor equations, such equations automatically hold with respect to any system of curvilinear coordinates.

\section{Einstein equivalence strong principle is false.}

In the search of generalize the Special Relativity, between October and November 1907, Albert Einstein adopted the equivalence principle (EP), consequence of the physical fact accepted by Isaac Newton as base of your mechanics, in particular with respect to the gravity (second law of the motion and law of universal gravitation), and originally principle of Galileo Galilei, its discoverer, ie: "Gravity accelerates everything object equally regardless of their masses or the materials from which they are made". The root cause of this principle is the equality of inertial and gravitational masses established in the Eötvös experiment.

"This principle was the rock upon which Einstein built his gravitation theory: in nine years of work on gravitation between 1907 and 1916, the principle of equivalence was, above all, the idea Einstein refused to give up, and yet in his very first statement of the principle he finds it inconsistent with the constancy of the velocity of light and with any generalization of the principle of relativity. The velocity of light cannot be the same in accelerated and unaccelerated frames; the laws in an unaccelerated frame may have to incorporate a homogeneous gravitational field which would vanish in an accelerated frame (Earman and Glimour, 1978) [12]. 
Einstein deduced further that gravity does not exist with respect to a freely falling system of reference and he raised to the which he called the Equivalence Principle between gravity and inertia, this is the weak equivalence principle (WEP). Too, Einstein extended EP to accelerated motion and gravity when he deduced that an accelerated system by a change of coordinates is interchangeable with a gravity system, today: Einstein equivalence strong principle (EEP) that says: "acceleration and gravity are equivalents" in terms of only one gravitational field, as is the case of the General Relativity, but which in the Brans-Dicke theory or in any other metric theory of gravity, is the strong equivalence principle (SEP).

Einstein reworked EP in EEP like the foundation that spacetime is curved, successfully supported in the gravitational redshift experiment (Clifford, 2006) [19], and he during the rest of your life insisted in the fundamental importance of this principle in the General Relativity, that nevertheless, it in its original formulation is not hold (Norton, 1985) [20].

The union of WEP and EEP produces that inertia, gravity and accelerated motion are equivalents, which implies: the nature laws are same in any reference systems with independence of their motion. This nice proposition is true only for homogeneous gravitational fields as are the previous to November 1915, when Einstein obtained the equations of General Relativity valid in curved spacetimes, therefore, for no-homogeneous gravity fields.

Although, EP was formulated during the Special Relativity, Einstein believed that too EP is valid in General Relativity because the homogeneous gravitational field is the particular case while no-homogeneous gravity fields is the case general, this is, the inertia is the particular case of the gravity. But, today, it is well established that "the equivalence principle, if understood as the possibility of excluding the gravitational field in an infinitesimal region, is not correct since there is no way in which we can exclude the curvature of space (if it is nonzero) by selecting an appropriate reference frame, even with in a given accuracy (Logunov and Mestvirishvili, 1986) [21].

In General Relativity, in a gravity field, the geodesic is curved and can not be overridden by any coordinate transformation (except at one point, but at one point is not possible do experiments, and although the laws that determine the structure of the tangent space are those of Special Relativity, the tangent space is not the the original space of reference).

Moreover, gravitational fields and fields of forces of inertia do no have a similar effect on all physical processes (Logunov and Mestvirishvili, 1986) [21], for example, in an accelerated system may appear electromagnetic waves: if inside the elevator Einstein is done empty then "at the time when the acceleration begins, the cabin floor emits an electromagnetic wave, which goes to the roof and oscillates back and forth, that William Unurh showed in 1976" (De Witt Bryce,1984) [22] or a charge in free fall according to electrodynamics must radiate energy, but due to the principle of equivalence between gravity and inertia, the charge is at rest in space and, therefore, should not radiate, however, the charge actually radiates (Baryshev, 2008) [23] etc.

“The validity of Einstein's strong version of the equivalence principle has been (and continues to be) challenged by many authors, usually based on the claim that tidal effects and/or intrinsic curvature make it possible to distinguish gravity from acceleration, even over arbitrarily small regions of spacetime. One of the most outspoken critics was J. L. Synge, who famously wrote (in his 1960 text on General Relativity): I have never been able to understand this Principle... Does it mean that the effects of a gravitational field are indistinguishable from the effects of an observer's acceleration? If so, it is false. In Einstein's theory, either there is a gravitational field or there is none, according as the Riemann tensor does not or does vanish. This is an absolute property; it has nothing to do with any observer's world-line. Spacetime is either flat or curved... The Principle of Equivalence performed the essential office of midwife at the birth of General Relativity, but... I suggest that the midwife be now buried with appropriate honors and the facts of absolute space-time faced...Clearly Synge was a proponent of the idea that gravity is to be identified with curvature, an idea which, as discussed above, is fundamentally contrary to the meaning and content of General Relativity" (Brown, 2015) [24].

The equivalence between acceleration and gravity is a fallacy that undermines the General Relativity theory. "It is shown that the real equivalence in local physical phenomena is between the effects of gravitational potential energy and those of kinetic energy" (Hatch, 2007) [5].

On other hand, Anatoly Logunov and M. Mestvirishvili proved that "the energy of a system and, therefore, the inertial mass of the system, have no physical meaning, since its magnitude depends on the choice of the coordinate system of three dimensions" (Logunov and Mestvirishvili, 1986) [21]. Indeed, a basic requirement of any definition of inertial mass is the independence of its magnitude of the choice of the coordinate system, which is valid for any physical theory. But, in General Relativity relativity the definition of inertial mass not meet this requirement, because "the definition of gravitational mass if it meets. "Since the inertial and gravitational mass obey to different laws of transformation, it violates the equality between the two masses"." Therefore, the claim that the inertial mass is equal to the gravitational mass in General Relativity has no physical meaning" (Logunov and Mestvirishvili, 1986) [21].

The gravity is truly a force of Lorentz, i.e, a force of quantum nature as the electromagnetic, strong and weak forces. So, the equivalence principle is false of root because the gravity and inertia are not the same; neither the gravity and the accelerated motion are not same. Gravity is a real physical force, while inertia is a effect of an reference system in uniform rectilinear motion with respect of other system taken at rest that by an appropriate change of coordinates these systems are interchangeable. Neither, gravity is equivalent to accelerated motion, because the gravity is a particular case of the accelerated motion, that is, when the force that to a mobile accelerates is the gravity force.

Although in Special Relativity EEP is accepted as true without objections, the author have showed that it is also false in Special Relativity. Two bodies, aligned according to the perpendicular, due to the reciprocal action of their gravity, the two bodies fall with different acceleration while in a gravitational system, they fall with equal acceleration (Guillen, 2014) [25].

Finally, both VLBI (Very Long Baseline Interferometry) and GPS (Global Positioning System) indicate that earth-based 
clocks are biased as a function of their position in the direction of the earth's orbital velocity. The evidence for these biases is confirmed by comparison of earth-based clocks with millisecond pulsars. These clock biases are precisely such as to cause the speed of light to appear as "c" in the earth's inertial frame. This shows that the speed of light is not isotropic in the earth's frame and that the Lorentz transformation is only an apparent transformation that results from Selleri's inertial transformations combined with clock biases (Hatch, 2004b) [4].

Too, clocks on the earth do not seem to be affected by the sun's gravitational potential. The most commonly accepted reason given is a faulty application of the equivalence principle. While no valid reason is available within either the special or general theories of relativity, ether theories can provide a valid explanation. A clock bias of the correct magnitude and position dependence can convert the Selleri transformation of ether theories into an apparent Lorentz transformation, which gives rise to an apparent equivalence of inertial frames. The results indicate that the special theory is invalid and that only an apparent relativity exists (Hatch, 2004a) [3].

\section{Mach's principle abandoned since 1918.}

The Mach's principle is the hypothesis that the inertial reaction forces experienced by bodies, when they are accelerated by external forces, are generated by action of the most distant matter in universe. The basic idea of this principle was stated by George Berkeley nearly 200 years before.

Mach's idea, about the relativity of inertia, was key in the building of General Relativity. Exactly, "Einstein gave the name of Mach's principle to the following related ideas: that only relative motion is observable, and hence that there should be no dynamically privileged reference frames; that inertial forces should arise from a gravitational interaction between matter only, and so from an observer-dependent splitting of the total gravitational field; that spacetime is not an absolute element in physics, but that its metric structure is totally dependent on the matter content of the universe" (Raine, 1975) [26].

According to the principle of general relativity, Einstein searched an explanation of inertia which would eliminate the privileged local inertial frames in Newtonian mechanics, therefore "based on that the measurements should not depend on the choice of coordinates assigned to events" (Lichtenegger and Mashhoon, 2008) [27]. But, "It has been shown that the relativity of all motion, i.e. the relativity of inertial accelerations, cannot be maintained in General Relativity. Starting from the assumption that inertial forces are of gravitational origin, neither translational nor rotational acceleration is compatible with the relativistic theory of gravitation, since the latter is based upon local Lorentz invariance and thus the notion of absolute motion cannot be avoided" (Lichtenegger and Mashhoon, 2008) [27]. "Although in General Relativity spatial geometries are affected by mass distributions, the geometry is not uniquely specified by the distribution. It has not yet been possible to specify boundary conditions on the field equations of General Relativity which would bring the theory into accord with Mach's principle. Such boundary conditions would, among other things, eliminate all solutions without mass present." (Brans and Dicke, 1961) [28].

In the context of the General Relativity, Einstein understood Mach's ideas on inertia through various ways "only arriving at his 1918 conception after failing adequately to cash out Mach's ideas in other ways in the years from 1912 to 1917; and that Einstein had to abandon this 1918 formulation of Mach's principle by the middle of that year"..."The most important differences between Einstein's understanding of Mach's principle in the 1913-1915 period, and the 1918 formulation, are two: first, in the early period there is, apparently, no recognition of the fact that an empty spacetime with Minkowski structure is incompatible with Mach's principle; and second, Einstein substantially equated general covariance, Mach's principle, and the equivalence principle. This equation was responsible for several conceptual problems that plagued Einstein prior to November, 1915, and also helped shape the next stage of Einstein's thinking on Mach's principle, in 1916" (Hoefer, 1995) [29]. "Inertial forces of all kinds are produced by interaction with other masses, and hence are just gravitational forces. On other hand is an extension of the equivalence principle. But as Minkowski spacetime is the most clearly anti-Machian spacetime possible and this is a solution of the Entwurf equations Einstein had leave. However the extension, in 1916, in the General Relativity theory, of the validity of the equations to all reference frames is just achieved by general covariance although is purely formal requirement of this, unrelated to the equivalence principle" (Hoefer, 1995) [29].

In the debate of 1916-18, at Leyden, between Einstein and Willem de Sitter, on the relativity of inertia, arise "the first two relativistic cosmological models: the Einstein cylinder world, filled with a uniform static mass distribution; and the completely empty De Sitter hyperboloid world (a name introduced in Weyl, 1923). In discussing the latter, Einstein and De Sitter had difficulty distinguishing features of the model from artifacts of its various coordinate representations. The situation was clarified in 1918 in correspondence between Einstein and two of the greatest mathematicians of the era, Hermann Weyl in Zurich and Felix Klein in Göttingen" (Janssen, 2010) [30].

Einstein said "first, that the universe is static; and second, that its metric structure is fully determined by matter i.e, that its metric field satisfies the Mach's principle. But, De Sitter's vacuum solution of Einstein's field equations with cosmological term is a counterexample to this principle. Einstein tried to discard it with the two arguments: one was that the De Sitter solution is not static; the other was that it has what today would be called an intrinsic singularity, which in turn was used to argue that it is not matter-free. In the end, Einstein had to acknowledge that the solution is fully regular and matter-free and hence indeed a counterexample to Mach's principle, but he could still discard the solution as physically irrelevant because it is not globally static" (Janssen, 2010) [30].

However, "Klein had shown that in the static form of the De Sitter solution, the time coordinate breaks down on the equator. In Weyl's hybrid static solution, on the other hand, which coincides with the De Sitter solution outside a zone of matter around the equator, the time coordinate is well defined everywhere. Only this hybrid solution, therefore, provides an 
acceptable static cosmological model. Einstein thus had to accept that the De Sitter solution forms a counterexample to Mach's principle as he had formulated it in March 1918. His modified field equations did allow fully regular matter-free solutions. He could still hold, however, that they did not allow globally static fully regular matter-free solutions. From a letter from Weyl to Klein of February 1919, written after consultation with Einstein, it can be inferred that this is the position to which Einstein retreated in response to Klein's analysis of the De Sitter solution. Although Einstein, in a letter of December 1918 to his friend Paul Ehrenfest, one of De Sitter's colleague in Leyden, expressed his regret that he had unjustly criticized De Sitter, he never published a correction to his critical note on the De Sitter solution" (Janssen, 2010) [30].

Local inertial frame is the one idealized as constituted by bodies on which no external forces act, with respect to which the very distant matter appears non-rotating although this matter is rotating truly. Empirically, this distant matter "coincides with the average system of the fixed stars, however, this correspondence appears incidentally, since the presence of the distant masses did in no way enter the calculations" (Lichtenegger and Mashhoon, 2008) [27].

Galileo was which introduced the concept of inertia of matter. He stated that a body, at absence of forces, remains at rest or is in a state of uniform rectilinear motion. Galileo established that the external forces are not cause velocity but of acceleration of bodies. And Newton quantified, in his second law of motion, that it applies only to inertial frames, that the acceleration of body is proportional to force. The inertial mass is the constant of proportionality and measures the inertia of body. The inertial forces locally may be interpreted as gravitational effects having their origin in distant matter accelerated respect local place.

When motion is relative to a non-inertial frames, it need of inertial forces to describe it. In the circular motion of an only body is possible establish absolute circular motion. 'The effects which distinguish absolute motion from relative motion are the forces of receding from the axis of circular motion. For there are no such forces in a circular motion purely relative, but in a true and absolute circular motion they are greater, or less, according to the quantity of the motion.' (Newton, 1686) [31]. But, if circular motion is of a water-filled bucket suspended from a twisted thread relative to a specific reference frame, the surface of water becomes concave (Newton, 1687) [32]. This is an absolute effect and can be used to measure the amount of rotation relative to that frame. A suggestion, not an explanation, was made by Mach, to the effect that inertial forces are related to the distant parts of the universe for the very good reason that they are generated by the distant parts of the universe. Mach did not give any quantitative theory for this somewhat vague concept, which later came to be known as Mach's principle" (Hoyle and Narlikar, 1963) [33] introduced by Einstein. In General Relativity, he succeeded in constructing a theory according to which accelerated and rotational motion is relative. If all motion is relative, the inertia of a body only it can measure with respect to something else. To the solutions of the equations in an universe of one body at the spacetime of Schwarzschild or in absolute motion at the spacetime of Kerr, Mach's principle would interpret such universe as no realist.

As the coupling to mass is gravity, General Relativity supposes the gravity is the source of inertial reaction forces (Woodward and Mahood, 2000) [34] and that the mass distributions affect the spacetime geometries. But. the General Relativity, like all other field theories, is a local field theory however, according to the Mach's principle, Einstein fixed the sources of the gravitational field at the global scale. The result is that inertia depends upon a mutual action to distance of matter that he called it "the relativity of inertia". However, the problem is that in the global solutions of the field equations the geometry is not uniquely specified by the mass distributions and "do not satisfy any reasonable formulation of, as he called it, Mach's principle. Even the addition of the cosmological constant term to his field equations didn't suffice to suppress the non-Machian solutions (Woodward, 2012) [35]. The anti-Machian character of the field equations, it arises of that is not possible to specify boundary conditions without mass present, and in particular, it shows up in solutions which allow for a curved spacetime even in the absence of any matter, as well as in solutions that exhibit an intrinsic rotation of matter with respect to the local inertial system (Lichtenegger and Mashhoon, 2008) [27]. "The fact that a local Galilean frame of reference or a local instrument such as the gyrocompass preserves its orientation with respect to the universe of stars is adequately explained by the fact that the universe of stars has the unique property of having no detectable mechanical interaction with local bodies... the universe of stars appears to have some connection with local phenomena for the paradoxical reason that it has no connection" (Bridgman, 1960) [36]. "Notably the curved space-time manifests itself also in rotational motion that does not appertain to the system itself. For example, the anomalous part of the perihelion precession of a planet does not ascribe to the solar system. Likewise, the axis of an onboard gyroscope of a satellite does not stay aligned with a distant star but will gradually precess in the orbital plane as well as in the equatorial plane of a revolving central body" (Annila, 2012) [37].

Mach, before his death in 1916, disavowed any association with Einstein's ideas on relativity and inertia. "Mach didn't suggest any effective relation between inertia and other stellar masses; but if we drop the particular philosophical context where the argumentations are suggested, a such link is easily deducible and this is the case for Einstein" (Gasco, 2004) [38].

In accordance with the principle of Mach, the following very weak effects should occur, difficult to measure (Einstein, 1955) [39] :

1. Inertia of a mass must increase, when this mass is together to other stacked masses, due to change of the potential gravitational energy to mass, consequence of $E=m c^{2}$. Einstein was wrong because only nongravitational energies contribute locally to $\mathrm{E}$.

2. A mass will accelerate, in the same direction, when other nearby masses are accelerated. Also, Einstein was partially wrong because this linear accelerative frame dragging, result of the general principle of relativity, applied 
to linear momentum, only it occurs when the universe is accelerated in any direction, therefore, only accelerations relative to the universe could be detectable (Woodward, 2012) [35].

3. A rotating hollow mass will generate a Coriolis field within the hollow, that can be explained as a Lens-Thirring effect or gravitomagnetic effect analog of electromagnetic induction. In the data from GP-B was said by group at Stanford University of NASA the frame-dragging effect was confirmed with an error of about 19 percent, and Einstein's predicted value was at the center of the confidence interval. However, the error is high. Also, "one remains puzzled out what exactly is the mechanism by which distant stars exert effects on local motions" (Annila, 2012) [37]. It can demonstrate quantitatively that the gravitomagnetic gyroscope precession within General Relativity is in conflict with Mach's principle. The conflict between General Relativity and Machian dragging can be clearly seen in the gravitomagnetic clock effect. Consider a circular equatorial geodesic orbit about a central rotating mass and imagine two free test clocks on this orbit moving in opposite directions. According to General Relativity, the clock in pro-grade motion moves slower and takes longer than the clock in retrograde motion to complete the orbit (Lichtenegger and Mashhoon, 2008) [27]. In 1922, Einstein was not satisfied with this third principle and said: "This contentedness will appear incomprehensible to a later generation however." ....."It must be said that, as far as I can see, to this day Mach's principle has not brought physics decisively farther. It must also be said that the origin of inertia is and remains the most obscure subject in the theory of particles and fields. Mach's principle may therefore have a future - but not without the quantum theory." (Pais, 1982) [40].

On other hand, author has proposed that in the exterior space the frame of motion has a physical reality, because it is the quantum vacuum. Therefore, this frame allows test the absolute motion, by the Doppler Effect induced by means of a decelerator of photons, affecting an electromagnetic wave in the direction of motion of an inertial system. This Doppler does not exist in the nature because the source of the electromagnetic wave is in rest respect to system. But, in each cycle, due to which the wave propagates inside the decelerator, the speed of wave $c^{\prime}$ combined with the speed of system $\mathrm{S}\left(\mathrm{s} \pm \mathrm{c}^{\prime}<<\mathrm{c}\right)$ respect to vacuum, provokes the induced Doppler. The result is that an observer, inside an inertial system, fully insulated from the outside, may determine whether his system is in rest or in motion and measure its speed, in absolute terms (Guillen, 2013) [41].

\section{The Einstein-Grossman equations.}

The Einstein-Grossmann equations are equations of field that generalize through of the tensor metric, the Newtonian potential gravitatory, and of the tensor of energy-impulse, the Newtonian mass, of the Poisson equation, without action at a distance and a dynamic spacetime, whose metric is the field. Since 18 November 1915, these equations were adjusted to give, in the equations of motion, the perihelion motion of the planet Mercury.

Grossmann was the one who led Einstein to use Riemannian spaces and the tensorial calculus developed by the end of the previous century by Gregorio Ricci-Curbastro. Too, Grossmann said to Einstein that the Riemann tensor curvature is the only tensor which can be formed from the metric tensor and their first and second derivatives, and further, that the contraction of the tensor, ie, the Ricci tensor, would be the only tensor for take the place of gravitational term and hold the general covariance.

The metric tensor simultaneously presents the static gravitational field and the structure of the spacetime (because the spacetime metric is the field) depending of dynamic equations that make it depends on the static gravitational field and its sources, that is, the metric itself is a dynamical object (spacetime constitutes both the dynamical object and the context within which the dynamics are defined).

The Einstein-Grossmann equations do not satisfy general relativity, equivalence and Mach principles of the General Relativity theory which only they were used as heuristic guidelines, but whether the general covariance, which is a tensorial property and no a principle. On other hand, the heuristic guidelines are inconsistent. However, these equations give exact outcomes, in the following tested cases:

1. The deflection of the light when it approximates to the Sun and, in general, of the gravitational lenses, consequence of the curvature of the spacetime around of the great masses. In 1976, Formalont measured with 1 percent accuracy the bending of radio waves emitted from a quasar when it approached eclipse by the Sun (Bruckman and Esteban, 1992). Also, bending of light ray passing near a massive elliptical galaxy or star, between spherical and elliptical shape objects, which led to appear a small but not negligible coefficient as oblateness independent of mass but depend on shape and geometry of object (Nikouravan and Rawal, 2013) [42].

2. The gravitational time dilation that is inverse to the gravitational potential (altitude respect to the center of the source of a gravitational field). The lapse measured by a clock is lower relative to a greater potential and potential greater inversely to the distance respect to center of the mass that generates it. The gravitational time dilation has been confirmed by the Gravitational redshift measured first time in the Pound-Rebka experiment.

3. Gravitational redshift of light produced by the gravitational dilation of time on the electromagnetic waves, that is the reduction of its frequency when they travel between a gravitational greater potential to a lesser potential, at the contrary case due to increase of the frequency, it produces the gravitational blue-shift. The frequency or wavelength shift between two identical frequency standards (clocks) placed at rest at different heights in a static gravitational field. The first successful, high-precision redshift measurement was the series of Pound-RebkaSnider experiments of 1960 - 1965. Until 2006, the most precise standard redshift test to date was the VessotLevine rocket experiment that took place in June 1976 at $10^{-4}$ level. The gravitational redshift could be improved 
to the $10^{-10}$ level using an array of laser cooled atomic clocks on board a spacecraft which would travel to within four solar radius of the Sun (Clifford, 2006) [43]. In 2012, this test has been improved to the $10^{-6}$ level (Guena and others, 2012) [44].

4. The Shapiro time delay that is direct to the curvature of a given spacetime, due to that the waves and particles travel a path more long and they use a greater time. This delay is measured respect to time used in a flat spacetime, i.e., Minkowskian. Using the Viking landers on Mart yielded an agreement with Einstein's theory with a impressive accuracy of 0.1 percent (Bruckman and Esteban, 1992) [45] .

5. The geodetic precession is produced in general in the spinning bodies in free fallen and in particular in the planets and is described as the very slow change in the direction of its rotational axis. In the long time, it traces, with respect to south and north poles, two cones (26.000 years in the case of Earth). In absence of the geodetic precession, planets would be repeating same orbit, i.e, same geodesy, but as geodesy is curve, the rotational axis after it has completed one orbit, its direction differs ever so slightly from the way it started, this is a property of the parallel transport on a curve (Einstein online). In the solar system, a part of precession of the orbit of a planet is caused by the gravity of the others planets (combined effects of the gravitational fields of the planets in the curvature of the global spacetime) and the rest, called anomalous precession of the perihelion of the orbits of the planets, by the curvature of the spacetime caused by sun. The curvature of space changes the way that our planet's spin axis precesses - these changes are very small and hard to measure (NASA, 2000) [46], since in a flat spacetime the direction of the rotational axis is constant in the time. In 2011, the geodetic precession, according Einstein, was confirmed at an accuracy of $0.28 \%$, in the GP-B prove (Everitt and others, 2011) [47].

6. The spacetime vortex is produced by the twisted of spacetime around of a planet due to its rotation. In general is called the interior gravitomagnetism the spacetime-dragging by the rotation of a planet. Also, it produces the exterior gravitomagnetism that is the spacetime-dragging by the orbital motion of a planet. In 2011, the interior gravitomagnetism, according Einstein, was confirmed at an accuracy of $19 \%$, in the GP-B prove (Everitt and others, 2011) [47].

7.The gravitational waves, that are the transversal propagation of undulations of the curvature of spacetime, are caused by asymmetric accelerations of binary pulsars or compound systems of a pair of white dwarfs, neutron stars, black holes e.t.c., also, in the gravitational asymmetric collapses, or asymmetric explosions in the nuclei of Galaxies, or asymmetric outbreaks of supernovas, or in the asymmetric sprouting of a stellar system or at the moment of the Big Bang, in that case called fundamental gravitational waves. In the systems of two masses, with time occurs variations of the common center of masses of system, this produces a quadrupole, that reunites the relation of the energy of four non-symmetrical angular moments of two masses. The gravitational waves carry energy, that in the lowest order is proportional to the quadrupole moment of the distribution of the mass-energy, in particular, of system of two masses. This radiation is originated in lost of the kinetic and/or potential energy, during the no uniform accelerations of the two masses or, in general, in the asymmetric accelerations. Thus, during the gravitational radiation, the mass in rest of the particles, constitutive of mass, does not change. Neither, the curvature of spacetime is source of energy carried by the gravitational waves. They deform the geometry of the spacetime, still more, and therefore, they produce more gravity, since they are nonlinear waves. The gravitational waves cause that the distances between the particles are changing over time. Sometimes, the gravitational wave stretches all vertical distances between particles and, at the same time, squeezes all horizontal distances. At other times, all horizontal distances are stretched while all vertical distances are squeezed (Einstein online). On the existence of the gravitational waves exist the binary pulsar B1913+16 that was the first to be discovered by Hulse and Taylor in 1975. The measured rate of change of orbital period agrees with that expected from the emission of gravitational radiation, according to General Relativity to within about 0.2 percent (Weisberg and Taylor, 2005) [48].

GP-B supposes that spacetime is a viscous fluid, therefore, both, the geodetic precession and the interior gravitomagnetism have been tested from false conceptual frame. Course, this is not the spacetime of General Relativity but spacetime in some alternative theories. However, this fundamental inconsistency is absolutely ignored.

\section{Conclusions.}

Prior to the theory of General Relativity, it says that there was not a theory of gravity, because, the gravitational aether theory of Newton is speculative and independent of the mathematic model of gravity. Only, through his gravitational equations, Newton defined operationally gravity, as a central force whose source are the masses of the particles or bodies interacting, acting instantly at a distance, in an absolute space.

With General Relativity theory, it says that there is a theory on gravity but truly no there a theory on gravity due to which between the principles of the theory and the field equations the link is only heuristic and is not structural, furthermore the principles are inconsistent because there are evidences to dispute them. On other hand, the General Relativity theory lacks of conceptual definitions in a formal language on gravity and spacetime, that like Newton are operational definitions.

Although, Einstein considered gravity as the inertial effect of the accelerated spacetime Einstein was wrong, because Einstein distinguished between homogeneous (flat spacetimes) and no-homogeneous (curved spacetimes) gravity fields, when a homogeneous gravity field is truly a curved spacetime due to which everything accelerated spacetime is a curved spacetime. The difference between a homogeneous gravity field and a no-homogeneous gravity field is which in the first the curves are not affine while in the second the curves are affine (they cut at a point). 
Thus, the strong equivalence principle between gravity and accelerated motion no exists because gravity is a special case of the accelerated motion and the weak equivalence principle between gravity and inertial motion no exists because gravity is affine curved while inertial motion is rectilinear. There is no general relativity principle because the special relativity principle was not generalized to arbitrary frames of reference, neither Mach principle because the quantum vacuum is the background of motion which makes possible establish the absolute motion.

Furthermore, Einstein was very confused when he considered spacetime like the structural property of the gravity field, due to that it causes circularity between the notions of spacetime and gravity, since, followers of Einstein consider gravity as effect of the curvature of spacetime caused by the energy-impulse, derived directly from the field equations. And yet, no one knows: What is spacetime?.

Today as yesterday, no there is a theory on gravity, but whether the Einstein-Grossmann's gravity equations more powerful that Newton's gravity equations.

\section{Bibliography.}

[1] Norton, J. D. (1993). General covariance and the foundations of General Relativity:eight decades of dispute:Rep. bog. Phys. 56, 791-858. one

[2] Hawking, S. W and Penrose, R. (1996). The Nature of Space and Time:Princeton University Press

[3] Hatch, R. R. (2004a). Clocks and the Equivalence Principle: Foundations of Physics, volume 34, Issue11, p. 1725-1739

[4] Hatch, R. R. (2004b). Those scandalous clocks:GPS Solutions, Vol. 8, 2004, p. 67

[5] Hatch, R. R. (2007). A New Theory of Gravity: Overcoming Problems with General Relativity: Physics Essays, 20, pp. 83-100.

[6] Weinstein, G. (2014). Did Einstein "Nostrify" Hilbert's Final Form of the Field Equations for General Relativity? Tel-Aviv University

[7] Norton, J. D. (1995). Did Einstein Stumble: The Debate over General Covariance:Erkenntnis, 42 , pp.223-245

[8] Einstein, A. (1913). Einstein was dissatisfied with his "Entwurf" field equations:Letter to Lorentz, on August 14.

[9] Renn, J. (2013) . Einstein's path to General Relativity: Max Planck Institute for the history of science. IHES Paris.

[10] Engler, F. O. (2009). Über das erkenntnistheoretische Raumproblem," in Stadler, 107-145

[11] Norton, J. D. (1984). How Einstein found his field equations: 1912-1915: Norton, J. [1984]:Birkhauser, pp. 101-59

[12] Earman, J and Glymour, C. (1978). Lost in the Tensors: Einstein's Struggles with Covariance Principles, 19121916:Studies in History and Philosophy of Science, Part A, 9 (4). pp. 251-278

[13] Einstein, A. (1907). On the Relativity Principle and the Conclusions Drawn from It:Jahrbuch der Radioaktivität, 4, 411462

[14] Einstein, A. (1912). On the Theory of the Static Gravitational Field:Annalen der Physik, ser. 4, 38, 443-458

[15] Dieks, D. (2005). Another look at general covariance and the equivalence of reference frames:Institute for the History and Foundations of Science Utrecht University,

[16] Einstein, A. (1918). On the Foundations of the General Theory of Relativity:Ann. Phys. 55 240-44

[17] Pooley, O. (2010). Substantive General Covariance: Another Decade of Dispute:In M. Suàrez, M. Dorato, and M. Rèdei (Eds.), EPSA Philosophical Issues in the Sciences: Launch of the European Philosophy of Science Association,

Volume 2, Chapter 19, pp. 197-209, Springer

[18] Rovelli, C. (2001). Quantum spacetime: What do we know?: Callender C, Huggett N (eds) Physics meets philosophy at the Planck scale. Cambridge University Press, Cambridge, pp 101-122, http://arxiv.org/abs/gr-qc/9903045

[19] Clifford M. Will, "The Confrontation between General Relativity and Experiment”, Living Rev. Relativity, 9, (2006), 3. [Online Article]: cited [June 2013], http://www.livingreviews.org//rr 20063

[20] Norton, J. (1985), What was Einstein's principle of equivalence?. Stud. in Hist. and Phil. of Sci., 16, 594-598

[21] Logunov A and Mestvirishvili M. (1989). The Relativistic Theory of Gravitation.Mir Publisher Moscow, 15-16

[22] De Witt Bryce. S. (1984). Gravedad cuántica.

[23] Baryshev Y. V. () Energy-Momentum of the Gravitational Field: Crucial Point for Gravitation Physics and Cosmology. Russia.

[24] Brown, K. (2015). Controversies Over the Equivalence Principle:MathPages

[25] Guillen, A. (2014). Critical failure of the principle equivalence between acceleration and gravity:Journal of Advances in Physics Vol 4, No. 2, p 524-529 
[26] Raine, D. F. (1975). Mach's principle in General Relativity:Mon. Not. R. astr. Soc. 171, 507-528

[27] Lichtenegger, H, and Mashhoon, B. (2008). Mach's Principle:arXiv.physics/0407078v2

[28] Brans, C. and Dicke, R. H. (1961). Mach's principle and a relativistic theory of gravitation:Physical review, vol 124, N 3

[29] Hoefer, C. (1995). Einstein's formulations of Mach's principle

[30] Janssen, M. (2010). The Einstein-De Sitter Debate and Its Aftermath:Hsci/Phys 4121

[31] Newton, I. (1686). Principia.

[32] Newton, I. (1686). Philosophiæ Naturalis Principia Mathematica

[33] Hoyle, F. and Narlikar, J. V. (1963). Mach's principle and the creation of matter:Proceedings of the Royal Society of London. Series A, Mathematical and Physical Sciences, Vol. 273. No. 1352

[34] Woodward, J. F. and Mahood, T. L. (2000). Mach's principle, mass fluctuations, and rapid spacetime transport:California State University Fullerton

[35] Woodward, J. F. (2012). Making Starships and Stargates: The Science of Interstellar Transport and Absurdly Benign Wormholes:Springer Science \& Business Media.

[36] Bridgman, P. W. (1960). Significance of the Mach principle:Harvard University, Cambridge, Massachusetts.

[37] Annila, A. (2012). Probing Mach's principle:Mon. Not. R. Astron. Soc. 423

[38] Gasco, E. (2004). Mach's Principle: the original Einstein's considerations (1907-12):Congress of the Italian Physical Society

[39] Einstein, A. (1955). The Meaning of Relativity, 5th ed., Princeton University Press, Princeton, pp. 99-108

[40] Pais, A. (1982). Subtle is the Lord: the Science and the Life of Albert Einstein, pp. 287-288. (Quoted by permission of Oxford University Press, Oxford)

[41] Guillen, A. L. (2013). On The Absolute Motion in the Inertial Systems: International Journal of Fundamental Physical Sciences (IJFPS), Vol 3, No 3, pp 50-53

[42] Nikouravan Bijan and Rawal J. (2013). Detection of Light Near Elliptical Galaxies or Stars in General Relativity:The Indian Planetary Society(IPS).

[43] Clifford M. Will, “The Confrontation between General Relativity and Experiment”, Living Rev. Relativity, 9,

(2006), 3. [Online Article]: cited [June 2013], http://www.livingreviews.org//rr-2006-3

[44] Guena J, Abgrall M, Rovera D, Rosenbusch P, Tobar M, Laurent P, Clairon A and Bize S. (2012), Improved tests of Local Position Invariance using $\mathrm{Rb}$ and $\mathrm{Cs}$ fountains. arXiv.org

[45] Bruckman W and Esteban E. (1992). An alternative calculation of light bending and time delay by a gravitational field:Physics Department, University of Puerto Rico

[46] NASA. (2000). Precession in Curved Space "The Geodetic Effect":NASA.

[47] Everitt C, DeBra D, Parkinson B, Turneaure J, Conklin J, Heifetz M, Keiser G, Silbergleit A, Holmes T, Kolodziejczak J, Al-Meshari M, Mester J, Muhlfelder B, Solomonik V, Stahl K, Worden P Jr., Bencze W, Buchman S, Clarke B, Al-Jadaan A, Al-Jibreen H, Li J, Lipa J, Lockhart J, Al-Suwaidan B, Taber M and Wang S. (2011). Gravity probe b: final results of a space experiment to test General Relativity:Physical review letters.

[48] Weisberg J and Taylor J. (2005). The Relativistic Binary Pulsar B1913+16:Thirty Years of Observations and Analysis:Binary Radio Pulsars ASP Conference Series, Vol. 328. 